\title{
Frames e dicionários onomasiológicos: uma proposta na interface entre semântica cognitiva e lexicografia
}

\author{
Frames and onomasiological dictionaries: a proposal in the \\ interface between cognitive semantics and lexicography \\ Diego Spader de Souza \\ Rove Luiza de Oliveira Chishman
}

Universidade do Vale do Rio dos Sinos - UNISINOS

\begin{abstract}
Resumo: O objetivo do presente artigo é tecer algumas considerações iniciais a respeito de uma proposta teórico-metodológica situada na interface entre a Semântica Cognitiva e a Lexicografia. Tal proposta, atualmente sendo desenvolvida em tese de doutorado, busca a aproximação entre a teoria da Semântica de Frames, de Charles J. Fillmore, com a onomasiologia e com a produção de dicionários onomasiológicos. $\mathrm{A}$ onomasiologia, tema de grande importância para a Semântica Estrutural, pode ser abordada, sob nosso viés, a partir do conceito de frame semântico introduzido por Fillmore. O artigo apresenta uma discussão teórica, buscando mostrar o potencial da interface, bem como as formas através das quais a Semântica de Frames pode auxiliar a prática lexicográfica. Por se tratar de uma teoria semântica baseada em conhecimento enciclopédico, a Semântica de Frames oportuniza um modelo de dicionário onomasiológico que leva em consideração o papel da experiência e da estrutura conceptual na descrição semântica.
\end{abstract}

Palavras-chave: Semântica de Frames. Semântica Cognitiva. Onomasiologia. Lexicografia. Dicionário onomasiológico.

Abstract: The purpose of this paper is to make some initial considerations about a theoretical-methodological proposal inserted in the interface between Cognitive Semantics and Lexicography. This proposal, which is currently being developed in a doctoral dissertation, seeks the approximation of Frame Semantics, conceived by Charles J. Fillmore, with onomasiology and the development of onomasiological dictionaries. Onomasiology, a subject of great importance for Structural Semantics, can be approached, under our bias, from the concept of semantic frame introduced by Fillmore. The article presents a theoretical discussion, seeking to show the potential of the interface, as well as the ways through which Frame Semantics can aid lexicographical practice. As a semantic theory based on encyclopedic knowledge, Frames Semantics provides a model for onomasiological dictionaries that takes into account the role of experience and conceptual structure in semantic description.

Keywords: Frame Semantics. Cognitive Semantics. Onomasiology. Lexicography. Onomasiological dictionary. 


\section{Introdução}

La semántica tiene como rama científica la respetable edad de unos setenta años. Ha tenido hijas e hijos, hijas semasiológicas e hijos onomasiológicos. No doy, por otro lado, ninguna garantía en cuanto al sexo; en todo caso, la familia se porta bien [...] (Baldinger, 1970, p. 14)

Os conceitos de onomasiologia e semasiologia foram, ao longo do século XX, amplamente discutidos no campo da semântica lexical que derivou da chamada Semântica Estrutural, baseada fundamentalmente na teoria do signo linguístico de Ferdinand de Saussure. Grosso modo, a onomasiologia e a semasiologia representam dois métodos opostos - ainda que complementares - de análise lexical. A semasiologia parte da palavra e chega às suas significações, assinalando um campo de significações; a onomasiologia, por outro lado, parte de um conceito e chega às possibilidades de realização linguística desse conceito, constituindo um campo de designações. (BALDINGER, 1966).

Essas duas noções, quando aplicadas à Lexicografia, deram origem a uma vasta tipologia de obras de referência. $O$ modelo tradicional de dicionário, que conta com uma lista alfabética de palavras acompanhadas de seus significados, segue uma lógica semasiológica. A onomasiologia, por sua vez, possibilitou a criação de dicionários sistemáticos (ou thesaurus), dicionários de sinônimos, dicionários reversos, dicionários ilustrados, entre outros. (cf. STERKENBURG, 2003).

O presente artigo tenciona apresentar considerações iniciais sobre uma proposta teóricometodológica de aproximação entre a lexicografia onomasiológica e a Semântica de Frames, teoria concebida por Charles J. Fillmore (1982, 1985). Uma das principais hipóteses que caracterizam a teoria é a de que palavras funcionam como pontos de acesso para porções organizadas de conhecimento enciclopédico, ou frames. Nossa proposta, atualmente sendo desenvolvida em tese de doutorado, objetiva estabelecer bases para 0 desenvolvimento de dicionários onomasiológicos voltados para usuários leigos e orientados pelos princípios da Semântica de Frames.

Isso exposto, este artigo, que apresenta um caráter mais "ensaístico", se organiza da seguinte forma: a seção 2 apresenta a primeira parte da interface, discutindo brevemente o conceito de onomasiologia e o dicionário onomasiológico; a seção 3 aborda os postulados teórico-metodológicos da Semântica de Frames; a seção 4 articula a interface, mostrando como os frames semânticos podem se encaixar na lexicografia onomasiológica; por fim, a seção 5 apresenta algumas considerações parciais a respeito da proposta, assim como perspectivas para a continuação da pesquisa.

\section{A onomasiologia e o dicionário onomasiológico: breves considerações}

Baldinger (1966) aborda os métodos onomasiológico e semasiológico no contexto da evolução da ciência linguística ao final do século XIX e início do $X X$, quando se passou do som à palavra. $A$ Linguística, antes unidimensional - fazendo referência aos modelos histórico-comparativos -, passou a ser tridimensional. Tornou-se, assim, estrutural. A partir do impacto causado por Saussure e da disseminação de suas ideias, a Linguística não só se estabeleceu como uma ciência moderna e autônoma, mas também iniciou uma fase de estudos voltados à língua enquanto estrutura. Passou-se ao estudo do sistema linguístico, um sistema solidário de signos.

Os conceitos de semasiologia e a onomasiologia surgem nesse contexto histórico, no início do século $X X$. Inicialmente atraídas pela pesquisa histórica (ou diacrônica), a onomasiologia e a semasiologia floresceram no campo dos estudos estruturalistas (que seguiam a perspectiva sincrônica defendida por Saussure). Retomando os dois conceitos, a semasiologia segue um percurso que parte da palavra e chega ao seu significado ou significados; a onomasiologia, em contrapartida, parte de um conceito (que é mental e abstrato) para chegar às palavras que realizam esse conceito no plano 
linguístico. A partir disso, segundo Baldinger (1966), a onomasiologia implica desde o início uma preocupação de ordem estrutural.

Foi no campo da Semântica Estrutural, inclusive, que se desenvolveu a teoria dos campos lexicais (ou campos semânticos), de autoria de Jost Trier. O pressuposto central dessa teoria é o de que determinados grupos de palavras pertencem a campos semânticos (como escola, trabalho, transporte etc.). Para Ullmann (1964), a teoria de Trier fornece meios valiosos para se lidar com um problema que é, ao mesmo tempo, difícil e de muita importância, que é a influência da linguagem no pensamento.

Especificamente em relação à semasiologia, Baldinger (1966) apresenta considerações de cunho mais histórico, ainda que defenda que "[...] o estabelecimento dos campos semasiológicos é a tarefa central de qualquer léxico alfabético e sincrônico" (p. 13). Ao refletirmos sobre o significado de uma palavra, somos levados a pensar nos usos e nos variados contextos em que tal palavra é utilizada. Em um dicionário alfabético de ordem semasiológica, substitui-se contextos por uma definição. E essa definição, que é o que se chama de estrutura semasiológica da palavra, é de grande importância para a interpretação de qualquer um dos contextos que uma dada lexia possa apresentar.

Posto que ainda houvesse muito a se dizer sobre a estrutura semântica de uma determinada palavra, é fato que, primeiramente, nos voltaríamos à estrutura semasiológica. Conforme Pierre Guiraud (1972), toda palavra tem uma espécie de "nó semântico" que é mais ou menos denso, mais ou menos volumoso, que é cercado por uma auréola de associações secundárias, afetivas ou sociais. O nó é o campo semasiológico, é a definição. Todo o resto compõe o conjunto de contextos de usos pessoais.

Somente a semasiologia, portanto, permite, partindo de um grande número de contextos de uso, estabelecer as generalizações de uma significação específica. A semasiologia trata, assim, da passagem do periférico para o central, ou do específico para o genérico. Um dicionário semasiológico, organizado alfabeticamente, não pode se deter a cada uso particular que uma palavra possa apresentar.

O percurso onomasiológico, por outro lado, inicia com um elemento mais geral, que é o conceito, e parte em busca daquilo que é mais específico, ou melhor, dos itens linguísticos de um léxico em questão que se relacionam a esse conceito. A semasiologia e a onomasiologia examinam as duas microestruturas fundamentais do léxico de uma língua. É fato que a onomasiologia promete resultados mais inovadores, principalmente levando em consideração a relação intrínseca que mantém com aspectos sociais e pragmáticos dos fatos linguísticos. (BALDINGER, 1966).

Dessa forma, o autor defende que se tenha dois tipos distintos de dicionários: um que parte de uma listagem alfabética (ou fonológica) e que liste significados e um que parta de conceitos. Tanto o método semasiológico quanto o onomasiológico, portanto, possuem razão de ser.

Embora os conceitos de semasiologia e onomasiologia apresentem um papel fundamental no Estruturalismo europeu, essa distinção, segundo Grondelaers e Geeraerts (2003), dificilmente ganhou espaço na terminologia da Linguística norteamericana. Pesquisadores associados à Linguística Cognitiva, Geeraerts e Grondelaers propõem um modelo pragmático de onomasiologia, de forma a desvendar o que leva falantes a escolherem determinadas palavras para referir um dado conceito. Os autores (2003) partem do pressuposto de que essas escolhas dependem da saliência semasiológica e onomasiológica das palavras e seus significados, constituindo grupos de palavras mais possíveis e menos possíveis de serem associadas a determinados conceitos.

Ainda que o modelo concebido por Geeraerts e Grondelaers, assim como nossa proposta, se encontre inserido na Linguística Cognitiva, nós nos voltamos especificamente para a interface entre a Semântica Cognitiva e a Lexicografia. Cabe salientar que a onomasiologia designa um tema pouquíssimo explorado até o momento pelos estudos cognitivistas. Embora a relação com a Lexicografia já tenha dado 
bons frutos (cf. OSTERMANN, 2015), não há pesquisas que busquem uma aproximação com o dicionário onomasiológico, assim mostrando também a importância deste trabalho.

Com relação ao dicionário onomasiológico, Babini (2001) aponta para o fato de que foi concebido para dar conta das dificuldades que um usuário pode enfrentar ao consultar um dicionário tradicional. Ainda que a lista alfabética seja consideravelmente fácil de lidar tanto para quem produz o dicionário quanto para quem o utiliza (BERGENHOLTZ; TARP, 1995), é possível que certo usuário tenha uma ideia do significado da palavra que quer encontrar, mas não a sua forma. É possível, ainda, que ele esteja à procura de palavras relacionadas, que podem ser sinônimos ou antônimos, por exemplo.

Uma das vantagens do dicionário onomasiológico, conforme Hüllen (1999), é a de apresentar muitas palavras semanticamente relacionadas lado a lado, dando acesso a um inventário muito maior de unidades lexicais. Consoante Babini (2001), o dicionário onomasiológico tem o dever de resolver o problema inverso daquele de um dicionário semasiológico. Para uma determinada ideia ou conceito, o dicionário de orientação onomasiológica deve apresentar a relação de palavras que se associam a essa ideia ou conceito.

Babini (2001) lista seis aspectos da estruturação do dicionário onomasiológico: (i) pelo sistema nocional ou plano de classificação dos conceitos, apresentados no início da obra lexicográfica, (ii) pela classificação sistemática das entradas, (iii) pelo conteúdo semântico das entradas, (iv) pela sinonímia, (v) pela antonímia, ou (vi) por analogia. Esses aspectos dão origem a uma tipologia diversificada de recursos lexicográficos onomasiológicos.

Sterkenburg (2003) cita, primeiramente, aquilo que chama de dicionários sistemáticos. Pode-se dizer que esse é o tipo mais prototípico de dicionário onomasiológico, uma vez que as palavras são reunidas a um conceito, sendo ele parte de um sistema guarda-chuva de conceitos. Esse tipo de dicionário foi inspirado pela visão de que a realidade a nossa volta pode ser dividida e descrita a partir de ideias. Vale mencionar que os dicionários sistemáticos ficaram particularmente famosos sob o nome de thesaurus (STERKENBURG, 2003). Podemos citar, por exemplo, o Thesaurus of English Words, publicado por Mark Roget em 1852, e o Longman Lexicon of Contemporary English, publicado por McArthur em 1981. Para o a língua portuguesa do Brasil, vale mencionar o Dicionário Analógico da Língua Portuguesa, de Francisco Ferreira dos Santos Azevedo.

O segundo tipo de dicionário onomasiológico trazido pelo autor (2003) é o dicionário de sinônimos, que são desenvolvidos com o objetivo de que usuários possam encontrar palavras alternativas àquela em que pensaram, mas que carregam 0 mesmo significado. Dicionários desse tipo podem ser divididos em: (a) dicionário de sinônimos tradicional, que traz informações em texto-corrido e (b) dicionários de sinônimos taxonômicos. Os dois tipos são ordenados alfabeticamente. Os verbetes são, geralmente, a palavra mais comum ou neutra de uma cadeia de sinônimos.

O terceiro tipo de dicionário onomasiológico apresentado é o dicionário reverso. Dicionários reversos tem uma macroestrutura formada por uma lista de significados alfabeticamente organizados seguidos pelas palavras a que pertencem. Segundo Sterkenburg (2003), o problema com esse tipo de dicionário é a dificuldade que o usuário encontra para formular a definição que leva a uma palavra. $O$ usuário deve formular uma pergunta mais ou menos como um lexicógrafo faria.

O quarto e último tipo de dicionário onomasiológico que mencionamos é o dicionário ilustrado. Os dicionários ilustrados são aqueles em que os verbetes são os nomes daquilo que está ilustrado ou simbolizado via imagens. A organização dos dicionários ilustrados é inteiramente onomasiológica (não há listas alfabéticas). No caso das obras desse tipo, as ilustrações substituem as definições lexicográficas. 
Isso exposto, passemos agora à segunda parte da interface, a Semântica de Frames.

\section{A Semântica de Frames: uma abordagem enciclopédica do significado}

Concebida inicialmente por Charles J. Fillmore (1982, 1985) e ainda hoje desenvolvida por pesquisadores associados ao projeto FrameNet, da Universidade da Califórnia em Berkeley, a Semântica de Frames caracteriza um dos modelos teóricos mais distintivos do campo da Linguística Cognitiva como um todo.

Originária das "guerras linguísticas" das décadas de 60 e 70, como bem lembram Salomão, Torrent e Sampaio (2013), a Linguística Cognitiva surge, em parte, impulsionada pela insatisfação de um grupo de pesquisadores (dentre os quais é importante mencionar George Lakoff, Ron Langacker, Leonard Talmy, Gilles Fauconnier e o próprio Fillmore) com o papel secundário dispensado à semântica e à pragmática em paradigmas então dominantes, como a Gramática Gerativa e a Semântica Formal.

Centralizando os estudos ligados às questões do significado e do uso da linguagem a partir de uma visão experiencialista (a compreensão e a conceptualização do mundo se dá através da interação e da experiência diária humana), a Linguística Cognitiva faz florescer uma Semântica Cognitiva, cuja premissa central é a de que a estrutura semântica equivale à estrutura conceptual (CROFT; CRUSE, 2004; EVANS; GREEN, 2006). Em outras palavras, o conteúdo semântico das unidades linguísticas é o próprio conhecimento de mundo dos falantes.

A Semântica de Frames é uma das teorias mais emblemáticas do movimento cognitivista porque reflete muito claramente esse princípio. O conceito de frame semântico se refere à própria estrutura conceptual. Segundo Fillmore (1982), um frame designa um sistema de conceitos inter-relacionados, de forma que compreender um desses conceitos determina compreender o sistema na sua totalidade.
Na perspectiva da Semântica de Frames, as palavras são pontos de acesso para estruturas maiores, ou seja, para os frames. O significado de uma palavra, portanto, é o frame que ela evoca.

De acordo com Croft e Cruse (2004), para entender o significado de "garçom", acessamos todo um conjunto de conhecimento enciclopédico relacionado a restaurantes. Acessamos o frame como um todo, o que inclui saber também o que são conta, cardápio, cliente, chef etc.

A Semântica de Frames deu origem ao projeto FrameNet de Berkeley (https://framenet.icsi.berkeley.edu/). Inspirada pela WordNet (FELLBAUM, 1998) e idealizada por Fillmore junto a outros pesquisadores da Linguística e da Computação, a FrameNet é desenvolvida no ICSI (International Computer Science Institute) da Universidade da Califórnia em Berkeley e caracteriza uma base de dados on-line de descrição do léxico da língua inglesa com base em frames com base em corpus. Os dados da FrameNet são disponibilizados de duas formas: uma linguagem legível para humanos e uma legível para máquinas. O intenso trabalho de descrição linguística da FrameNet deu origem a dois conceitos muito importantes para a interface com a Lexicografia: o de elemento de frame e o de unidade lexical.

A noção de elemento de frame está presente desde os primeiros artigos de Fillmore em que ele apresenta os contornos do que viria a ser a Semântica de Frames, ainda na década de 70 . Contudo, o conceito só ganhou traços mais "teóricos" a partir do trabalho da FrameNet, em que os elementos de frame constituem uma das categorias para a descrição dos frames. Ao descrever uma festa de aniversário, poderíamos elencar, por exemplo, um aniversariante, um bolo, presentes, convidados etc. Esses seriam os elementos de frame de um frame Festa de Aniversário. São os sujeitos e instituições que compõem uma determinada cena social. (cf. BAKER; FILLMORE; LOWE, 1998).

O conceito de unidade lexical, por sua vez, não é próprio da teoria da Semântica de Frames, sendo característico do campo dos estudos lexicais como 
um todo. Entretanto, a partir da FrameNet, ganha contornos mais específicos. Segundo Baker (2014), tradicionalmente, o conceito de unidade lexical reflete a natureza do signo linguístico nos termos de Saussure, sendo o pareamento entre uma forma, ou significante, e um significado. Para a FrameNet, tratase do pareamento entre uma forma e um frame. Por exemplo, os verbos comprar e dever são formas linguísticas que evocam o frame Transação Comercial. (FILLMORE, 1982; PETRUCK, 1996).

O trabalho realizado pela FrameNet tem dado origem a projetos similares para outras línguas (multilingual framenets), como o português brasileiro (SALOMÃO, 2009), o espanhol (SUBIRATS, 2009), o japonês (OHARA, 2009), e o hebreu (PETRUCK, 2009), entre outras.

Além disso, cabe salientar o desenvolvimento de dicionários eletrônicos para domínios especializados baseados em frames, como o Field Dicionário de Expressões do Futebol (CHISHMAN, 2014) e o Dicionário Olímpico (CHISHMAN, 2016), ambos inspirados pelo projeto Kicktionary (SCHMIDT, 2009), bem como o FrameNet Brasil Copa 2014 (SALOMÃO, 2014). Diferente de dicionários tradicionais, impressos ou on-line, essas obras fornecem não apenas uma descrição das unidades lexicais baseada em frames, como também uma estrutura de acesso via frames, o que caracteriza um viés onomasiológico, uma vez que o usuário pode iniciar a consulta pelo conceito.

Contudo, ainda que exista, de certa forma, um aspecto onomasiológico presente nesses dicionários, nossa proposta é utilizar os pressupostos da Semântica de Frames para enriquecer a estruturação e a descrição de domínios onomasiológicos em dicionários específicos dessa orientação. Não se trata de propor um modelo de recurso lexicográfico baseado em frames, mas de estabelecer bases para uma onomasiologia que leva em consideração aspectos enciclopédicos; o frame é uma das possibilidades da Semântica Cognitiva para que se alcance esse objetivo.

Passamos agora à próxima seção, em que discutimos pontos da interface proposta.

\section{A proposta teórico-metodológica: breves considerações iniciais}

Nesta parte do artigo, dedicamo-nos, conforme já dito, a explorar alguns traços da proposta teóricometodológica que vem sendo desenvolvida em tese de doutorado. Por se tratar de um trabalho ainda em progresso, o que apresentamos aqui são considerações iniciais sobre o funcionamento da interface entre frames semânticos e lexicografia onomasiológica. Seguindo os passos dos dicionários baseados em frames já existentes, nossa proposta também se volta para o campo dos dicionários eletrônicos, que se encontra em rápida expansão. (cf. GRANGER, 2012).

Fillmore (2003), inclusive, já considerava que a Semântica de Frames seria melhor abordada pela Lexicografia em contexto on-line, uma vez que o conhecimento enciclopédico presente nos frames dificilmente poderia ser representado em dicionários impressos, dada a sua limitação de espaço. O meio eletrônico fornece a possibilidade de hiperlinks e espaço para uma vasta quantidade de dados.

Com relação à macroestrutura (estrutura de acesso, disposição dos dados, cf. ATKINS; RUNDELL, 2008) de um dicionário nos moldes da proposta, a Semântica de Frames aponta para a possibilidade de se utilizar o maquinário da Linguística de Corpus para a identificação das duas macroestruturas: o conjunto de domínios onomasiológicos (os frames) e as unidades lexicais. Consideramos dois tipos de corpora: um corpus de pesquisa, processável por softwares da Linguística de Corpus, e um corpus de estudo. $\mathrm{Na}$ tese de doutorado em desenvolvimento, a proposta teóricometodológica será discutida a partir do campo da gastronomia. Nesse sentido, serão compilados um corpus processável para a identificação das unidades lexicais e um corpus não-processável para a identificação dos campos onomasiológicos.

Outro ponto a ser destacado com relação à macroestrutura diz respeito à terminologia da Semântica de Frames. Ainda que, neste artigo, nos refiramos a frames e a elementos de frame, cabe 
salientar que a estrutura a que o usuário deve ter acesso não será composta por termos específicos à teoria.

Com relação à microestrutura, primeiramente, temos a estrutura interna dos domínios onomasiológicos, que poderá ser composta por: (i) uma glosa, (ii) elementos de frame, (iii) relações entre frames e (iv) palavras do frame. Os elementos de frame devem ser listados a partir do estudo dos domínios onomasiológicos. A glosa deve levar em consideração os elementos de frame e o conhecimento enciclopédico relacionado ao domínio. As relações entre frames levam em consideração os relacionamentos entre o frame e os outros domínios. As unidades lexicais são as palavras que evocam o domínio.

A microestrutura das unidades lexicais não ganha definição, o que comporia um traço semasiológico. Com isso, as unidades lexicais são compostas por: (i) indicação do frame a que pertencem, (ii) sentenças exemplo extraídas de corpus e (iii) relações semânticas entre palavras do domínio. A indicação do frame é bastante simples, basta apenas que se tenha um link para o frame evocado pela palavra. As sentenças exemplo, advindas do corpus de pesquisa, apresentarão um tipo simplificado de anotação semântica, apenas no intuito de marcar a unidade lexical e os elementos de frame. O desafio é apresentar essas sentenças exemplo de forma a não confundir o usuário leigo, não comprometendo a sua experiência. Por fim, as relações semânticas entre palavras constituem uma parte mais tradicional dos dicionários onomasiológicos, de forma que serão listadas as relações de sinonímia, polissemia e hipo/hiperonímia. Como essas relações serão trabalhadas ainda é um de nossos desafios.

\section{Considerações parciais}

O presente artigo teve dois objetivos principais: apresentar as duas partes da interface que compõem a proposta teórico-metodológica (a lexicografia onomasiológica e a Semântica de Frames) e discutir brevemente alguns pontos de articulação da interface, levando em consideração que a proposta se encontra ainda em desenvolvimento. Não se trata, portanto, de uma reflexão acabada.

No âmbito deste trabalho, buscou-se mostrar o potencial da Semântica de Frames para a Lexicografia, uma discussão que se enquadra numa problemática ainda maior, que é a da utilização de teorias semânticas na prática lexicográfica (cf. LEW, 2007). A Semântica de Frames, por representar um modelo teórico enciclopédico, se encaixa bem à onomasiologia, haja vista o caráter "extralinguístico" que lhe é intrínseco. A onomasiologia, ao iniciar pelo conceito e não pela forma, certamente enfrentaria dificuldades ao tentar se desvencilhar do que é enciclopédico. A Semântica de Frames - e a Semântica Cognitiva como um todo, cabe dizer -, ao dissolver as barreiras entre o que é linguístico e nãolinguístico, se apresenta como um modelo já onomasiológico em sua natureza: o frame é o ponto de partida.

Nesse sentido, a partir desta breve exposição, reiteramos o valor da Semântica de Frames para a Lexicografia e para a tarefa de propor bases teóricometodológicas para dicionários onomasiológicos. Em tempo, reiteramos também o papel central da Lexicografia Eletrônica, que, acompanhando os rápidos avanços tecnológicos, cada vez mais se firma no quadro da produção de dicionários.

\section{Referências Bibliográficas}

ATKINS, B. T. Sue; RUNDELL, Michael. The Oxford Guide to Practical Lexicography. New York: Oxford University Press, 2008. 540 p.

BABINI, Maurizio. Onomasiologie et dictionnaires onomasiologiques. São José do Rio Preto: Editora Beatriz, 2001.

BAKER, Collin F. FrameNet: A Knowledge Base for Natural Language Processing. Proceedings of Frame Semantics in NLP: A Workshop in Honor of Chuck Fillmore (1929-2014), Baltimore, jun. 2014. p. 1-5.

BAKER, Collin F.; FILLMORE, Charles J.; LOWE, John B. The Berkeley FrameNet Project. In: BOITET, Christian; WHITELOCK, Pete (Eds.). 36th Annual Meeting of the Association for 
Computational Linguistics and 17th International Conference on Computational Linguistics, COLING-ACL '98. Montreal: Morgan Kaufmann Publishers, 1998. p. 86-90.

BALDINGER, Kurt. Semasiologia e Onomasiologia. Tradução de Ataliba T. de Castilho. Alfa, São Paulo, v. 9, p. 7-36, 1966.

BERGENHOLTZ, Henning; TARP, Sven. Manual of Specialized Lexicography: The Preparation of Specialized Dictionaries. 1 ed. Amsterdan/Philadelphia: John Benjamin Publishing Company, 1995. 254 p.

CHISHMAN, Rove L. O. Field - Dicionário de Expressões do Futebol. São Leopoldo: UNISINOS, $2014 . \quad$ Disponível em: $<$ www.dicionariofield.com.br>. Acesso em: 16 jun. 2017.

CHISHMAN, Rove L. O. Dicionário Olímpico. São Leopoldo: UNISINOS, 2016. Disponível em: $<$ www.dicionarioolimpico.com.br>. Acesso em: 16 jun. 2017.

CROFT, William; CRUSE, D. Alan. Cognitive Linguistics. Cambridge: Cambridge University Press, 2004. $356 \mathrm{p}$.

EVANS, Vyvyan; GREEN, Melanie. Cognitive Linguistics: An Introduction. Edimburgo: Edinburgh University Press, 2006. 830 p.

FELLBAUM, Christiane (Ed.). WordNet: An Electronic Lexical Database. Cambridge: MIT Press, 1998. $425 \mathrm{p}$.

FILLMORE, Charles J. Frame semantics. In: The Linguistic Society of Korea (Eds.). Linguistics in the Morning Calm. Seoul: Hanshin Publishing Co., 1982. p. 111-137.

Frames and the semantics of understanding. Quaderni di Semantica, Alessandria, v. 6, n. 2, dez. 1985. p. 222-254.

GUIRAUD, Pierre. A Semântica. Rio de Janeiro: DIFEL, 1975.140 p.

GRANGER, Sylviane. Introduction: Electronic lexicography - from challenge to opportunity. In: GRANGER, Sylviane; PAQUOT, Magali (Eds.). Electronic Lexicography. Oxford: Oxford University Press, 2015. 517 p. p. 1-13.

GRONDELAERS, Stefan; GEERAERTS, Dirk Towards a pragmatic model of cognitive onomasiology. In: CUYCKENS, Hubert; DIRVEN, René; TAYLOR, John R. Cognitive Approaches to Lexical Semantics. Berlim/Nova York: Mouton de Gruyter, 2003. 502 p. p. 67-92.

HÜLLEN, Werner. The Onomasiological Approach. In: English Dictionaries, 800-1700: The
Topical Tradition. Oxford: Oxford University Press, 1999. 544 p. p. 3-27.

LEW, Robert. Linguistic semantics and lexicography: A troubled relationship. In: FABISZAK, Małgorzata (Ed.). Language and Meaning: Cognitive and Functional Perspectives. Berna: Peter Lang, 2007. 342 p. p. $217-224$.

OHARA, Kyoko Hirose. Frame-based contrastive lexical semantics in Japanise FrameNet: The case of risk and kakeru. In: BOAS, Hans C. (Ed.). Multilingual FrameNets in Computational Lexicography. Berlim/Nova York: Mouton de Gruyter, 2009. 352 p. p. 163-182

OSTERMANN, Carolin. Cognitive Lexicography. A New Approach to Lexicography Making Use of Cognitive Semantics. Berlim/Nova York: Mouton de Gruyter, 2015. 380 p.

PETRUCK, Miriam R. L. Frame Semantics. In: VERSCHUEREM, Jef; ÖSTMAN, Jan-Ola; BLOMMAERT, Jan. (Eds.). Handbook of Pragmatics. Amsterdam/Filadélfia: John Benjamin Publishing Company, 1996. 658 p. p. 1-13.

Typological considerations in constructing a Hebrew FrameNet. In: BOAS, Hans C. (Ed.). Multilingual FrameNets in Computational Lexicography. Berlim/Nova York: Mouton de Gruyter, 2009. 352 p. p. 183-207.

SALOMÃO, Maria Margarida Martins. FrameNet Brasil Copa 2014. Juiz de Fora: UFJF, 2014.

SALOMÃO, Maria Margarida Martins. FrameNet Brasil: um trabalho em progresso. Calidoscópio, São Leopoldo, v. 7, n. 3, p. 171-182, set./dez. 2009.

SALOMÃO, Maria Margarida Martins. TORRENT, Tiago Timponi. SAMPAIO, Thais Fernandes. A Linguística Cognitiva encontra a Linguística Computacional: notícias do projeto FrameNet Brasil. Cadernos de Estudos Linguísticos, Campinas, v. 55, n. 1, p. 7-34, jan./jun. 2013.

STERKENBURG, Piet van. (Ed.). A practical Guide to Lexicography. 1 ed. Amsterdam/Filadélfia: John Benjamin Publishing Company, 2003.

SUBIRATS, Carlos. Spanish FrameNet: A framesemantic analysis of the Spanish lexicon. In: BOAS, Hans C. (Ed.). Multilingual FrameNets in Computational Lexicography. Berlim/Nova York: Mouton de Gruyter, 2009. 352 p. p. 135-162.

ULLMANN, Stephen. Semântica: uma introdução à ciência do significado. Tradução de J. A. Osório Mateus. Lisboa: Fundação Calouste Gulbenkian, 1964. 


\section{COMO CITAR ESSE ARTIGO}

SPADER DE SOUZA, Diego; CHISHMAN, Rove. FRAMES E DICIONÁRIOS ONOMASIOLÓGICOS: UMA PROPOSTA NA INTERFACE ENTRE SEMÂNTICA COGNITIVA E LEXICOGRAFIA. Signo, Santa Cruz do Sul,

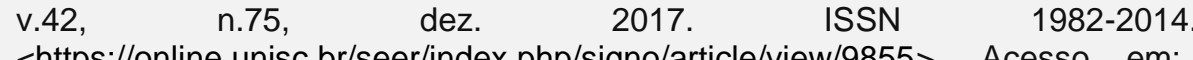
<https://online.unisc.br/seer/index.php/signo/article/view/9855>. Acesso em: doi: http://dx.doi.org/10.17058/signo.v42i75.9855. 